\title{
Análisis fenomenológico de la conciencia del docente a partir de sus prácticas evaluativas
}

\author{
Carmen García-Quintero ${ }^{\mathrm{a}}$, Gustavo Villamizar Suárez ${ }^{\mathrm{b} *}$

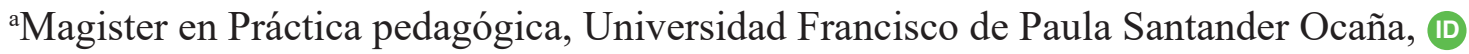 \\ boctor en educación, Universidad Francisco de Paula Santander (ID)
}

Forma de citar: Quintero, C. \& Villamizar, G. (2017). Análisis fenomenológico de la conciencia del docente a partir de sus prácticas evaluativas. Perspectivas, 2(2). 49-59.

Recibido: noviembre 12 de 2016

Aceptado: marzo 24 de 2017

\section{Palabras clave \\ Fenomenología, conciencia del docente, prácticas evaluativas}

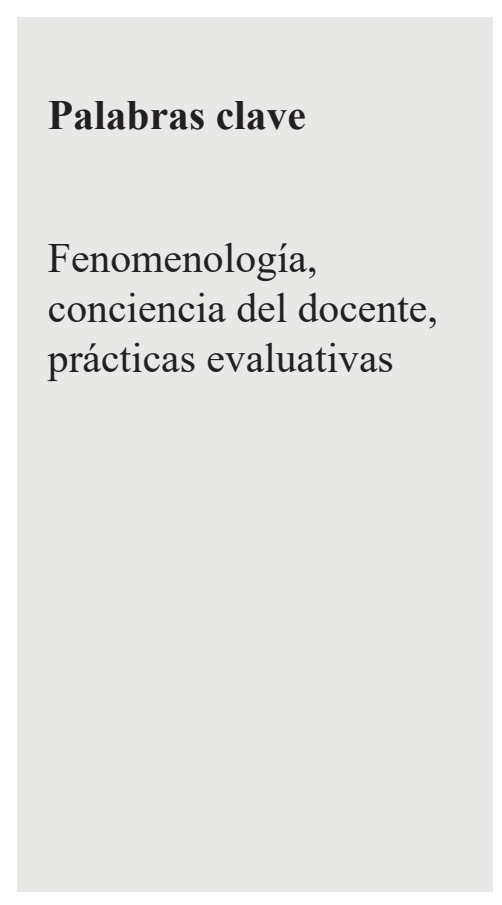

Resumen: Se analizan los cambios, logros y desarrollos esenciales que como expresión de la conciencia adquieren los docentes en su interacción con la práctica evaluativa. Esta lectura fenomenológica ayuda a comprender cómo los acontecimientos externos motivan acciones que se imponen y desencadenan internamente formas de subjetivación y señala qué acciones giran alrededor de la práctica evaluativa como reflejo de la conciencia que ha adquirido el sujeto evaluador. El método fenomenológico permite establecer las concepciones que sobre la experiencia evaluativa vivida a lo largo de años de formación de los sujetos, la convierten en parte sustancial de la conciencia tanto de docentes como de estudiantes. Se analiza fenomenológicamente cuál es la intencionalidad de las prácticas evaluativas desde la conciencia del docente universitario de la Universidad Francisco de Paula Santander. Se encontró que el discurso y la práctica evaluativa están enmarcados en enunciaciones de tipo autoritario, utilizado como dispositivos de poder, lo cual definir el rumbo o utilización que el sujeto evaluador hace de la evaluación.

\footnotetext{
*Autor para correspondencia gustavovs@ufps.edu.co

Universidad Francisco de Paula Santander, Cúcuta.
} 


\section{Keywords}

Phenomenology, teacher awareness, evaluation practices

\section{Palavras chave}

Fenomenologia, conscientização do professor, práticas de avaliação

\section{Phenomenological analysis of the teacher's conscience based on their evaluation practices}

\begin{abstract}
This paper presents and analyzes the changes, achievements and essential developments that, as an expression of consciousness, teachers acquire during their involvement with the assessment process. This investigation operates from a phenomenological perspective and attempts to discern how external events motivate actions that trigger forms of inward subjectivation, as well as highlights which actions are related to the assessment process as a reflection of the consciousness that the evaluative subject has acquired. The phenomenological method allows us to identify the conceptions about the evaluative experience (which involves years of individual training) that becomes it a substantial part of the consciousness of both teachers and students. The aims of the assessment process are analyzed phenomenologically, concerning the consciousness of the professors at the Francisco de Paula Santander University. It was found that the assessment process and the discourse surrounding it are framed in clearly defined statements, used as power dispositifs, which determine show the evaluating subject uses the evaluation.
\end{abstract}

\section{Análise fenomenológica da consciência do professor com base em suas práticas de avaliação}

Resumo: As mudanças, realizações e desenvolvimentos essenciais que os professores expressam em sua interação com a prática avaliativa são analisados como uma expressão de consciência. Esta leitura fenomenológica ajuda a entender como os eventos externos motivam ações que impõem e desencadeiam internamente formas de subjetivação e aponta quais ações giram em torno da prática avaliativa como um reflexo da consciência que o sujeito avaliador adquiriu. O método fenomenológico permite estabelecer as concepções que, ao longo da experiência avaliativa vivida durante anos de treinamento dos sujeitos, tornam a parte substancial da consciência de professores e alunos. É analisado fenomenologicamente qual é a intencionalidade das práticas avaliativas da consciência do professor universitário da Universidade Francisco de Paula Santander. Verificou-se que o discurso e a prática avaliativa estão enquadrados em enunciados de tipo autoritário, utilizados como dispositivos de poder, que definem o curso ou o uso que o sujeito avaliador faz da avaliação. 


\section{Introducción}

El presente trabajo se justifica desde el análisis fenomenológico de los tipos de cambio, logros y desarrollos esenciales que como expresión de conciencia adquieren los docentes en su interacción con la práctica evaluativa (Aguirre García y Jaramillo Echeverri, 2012). Ante las situaciones de evaluación cabe interpretar por qué el sujeto docente piensa y actúa de una determinada manera y no de otra manera y por qué estos sujetos realizan estas prácticas específicas (Careaga, 2001). Esta lectura fenomenológica consiste en una nueva mirada que ayuda a comprender cómo los acontecimientos externos motivan acciones que se imponen y desencadenan internamente formas de subjetivación y señala qué acciones, qué hábitos giran alrededor de la práctica evaluativa como reflejo de la conciencia adquirida por el sujeto evaluador (Moustakas, 1994; Fermoso, 2008).

En este sentido, este estudio se presenta como una opción de análisis para los docentes y sus instituciones y tiene como objetivo, analizar fenomenológicamente la conciencia del docente a partir de las prácticas evaluativas, estableciendo las acciones y formas de pensar que, como expresión de conciencia, realiza el docente desde la evaluación a través de la identificación de los discursos evaluativos que sustenta su manera de pensar y actuar desde su práctica evaluativa (Zuluaga, 2009; Torres, 2010).

\section{Materiales y métodos}

Esta investigación se centra en el paradigma cualitativo y el enfoque fenomenológico para la comprensión de las interacciones que se dan entre la conciencia del sujeto y su práctica evaluativa. Según Weeks (1984) el enfoque de la acción social o fenomenológico considera que la comprensión de los significados de las organizaciones y de los individuos que las integran no pueden obtenerse de forma independiente ni hacer generalizaciones sobre ellas, dado que la comprensión del significado se obtiene a partir de la percepción de la interacción entre ellos y los significados que los individuos dan a su experiencia.

De acuerdo con Martínez (2004), el objetivo del acercamiento fenomenológico, desarrollado por Husserl (Lambert, 2006; Anzola, 2007), es adquirir una comprensión de las estructuras esenciales de estos fenómenos sobre la base de ejemplos mentales proporcionados por la experiencia o la imaginación.

\subsection{Informantes clave y contexto}

Se seleccionaron docentes de planta vinculados a los programas académicos de la Facultad de Ciencias Agrarias y del Ambiente adscritos a los Departamentos Pecuario, Agrícola y Ambiental de la Universidad Francisco de Santander en Ocaña. A partir de un muestreo intencional se trabajó con ocho docentes. Simultáneamente se constituyó un equipo de observadores conformado por 16 estudiantes que cursan las asignaturas de cada docente observado.

\subsection{Instrumentos}

La información requerida para esta investigación proviene de dos fuentes: la primera sobre las acciones que el sujeto evaluador realiza en su práctica y la segunda sobre la forma de pensar que tiene el sujeto respecto a las acciones evaluativas.

Para la primera acción se constituyó un equipo de observadores conformado por 16 estudiantes que cursan las asignaturas de cada docente observado, los cuales llevaron a cabo el proceso de recolección de los datos sobre las acciones que el docente realiza alrededor de la práctica evaluativa en tres momentos: antes, durante y después de la evaluación. El total de horas de observación sobre las prácticas de los docentes participantes fueron 96. Los momentos observados y las observaciones registradas de acuerdo a los momentos fueron 88 .

Para la segunda acción sobre la forma de pensar de los docentes, se eligió la entrevista como 
la técnica. El instrumento se aplicó a una muestra de ocho docentes de la Facultad de Ciencias Agrarias y del Ambiente de la UFPSO, que identificaron en ellos los conceptos, las creencias y las afirmaciones que poseen sobre el proceso evaluativo con el fin de encontrarlos discursos que sustentan la práctica evaluativa que realizan.

\subsection{Procedimiento}

De acuerdo con Mollà, Bonety Climent, (2010), la secuencia a seguir en el análisis fenomenológico de los datos obtenidos en una entrevista es la siguiente: 1) transcripción; 2) elaboración de unidades de significado general; 3) elaboración de unidades de significado relevante para el tema de la investigación;4) verificación de las unidades de significado relevante y 5) conclusión.

A partir de los datos obtenidos mediante los instrumentos utilizados en esta investigación, se organiza el proceso requerido para abordar la conciencia del docente mediante el enfoque fenomenológico en las etapas siguientes:

Etapa previa: Clarificación de los presupuestos. Los presupuestos de los cuales se partió en la presente investigación fueron los siguientes:

1. La relación que permanentemente tiene el docente universitario con la práctica evaluativa genera en él un proceso de conciencia que desemboca en la conciencia misma.

2. El proceso de conciencia puede hacerse explícito en las acciones, pensamientos hábitos que en sí mismo manifiesta como una forma natural deser.

3. Estas acciones, pensamientos y hábitos de los que se apropia el docente en su interacción con la práctica evaluativa constituyen vivencias o modos conscientes de ver la realidad evaluativa.

4. Siendo estas acciones, pensamientos y hábitos indicadores de conciencia, solo pueden ser inferidos de la vivencia manifiesta, pues obedecen a un proceso consciente dentro de la subjetividad del docente.

5. Los docentes universitarios pueden expresar estas vivencias de manera consciente.

6. El docente universitario ejerce una acción voluntaria e intencional sobre el campo evaluativo al considerarlo como un objeto percibido y pensado.

7. Como consecuencia de su relación con la práctica evaluativa el docente universitario manifiesta la percepción de sí mismo.

Esta primera etapa fundamenta las bases para el logro del momento fenomenológico de la siguiente etapa, ya que el acto de explicitar estos presupuestos esenciales como paso previo, permite al investigador ponerlos entre paréntesis, practicar la epojé fenomenológica.

Etapa descriptiva: Descripciones protocolares. Se realizó la transcripción de los 88 momentos observables con los ocho docentes participantes del proyecto y las ocho entrevistas al instrumento de registro diseñado. Esta etapa del método fenomenológico consistió en hacer una primera descripción fenomenológica en el sentido de tener esas respuestas tal como ellos las habían expresado, sin ningún tipo de interpretación y análisis. En la descripción fenomenológica realizada como producto de esta etapa, se practicó una segunda reducción fenomenológica, en tanto que se pusieron ente paréntesis referencias no relacionadas con la manifestación de la práctica evaluativa quedando solo lo dado.

Etapa estructural. Habiéndose realizado las descripciones protocolares entre observación y entrevista de esta etapa estructural se procedió a su estudio y análisis con el fin de encontrar en ellas significados esenciales.

\section{Resultados y discusión}

La aplicación del procedimiento planteado, permitió concebir los distintos momentos y los pasos a seguir dentro de cada uno de ellos. Las 
reducciones fenomenológicas correspondientes a cada momento se exponen a continuación.

Primer paso. Se realizó una primera lectura de cada una de las descripciones protocolares, a fin de tener una primera visión del tema central al que cada una de ellas se refería.

Segundo paso. Se realizó una segunda lectura de las 96 observaciones para identificar en cada una de ellas el tema central. Esta segunda lectura se hizo respondiendo a los interrogantes: ¿qué es lo que el docente manifiesta en esta descripción protocolar?, ¿de qué trata esta descripción protocolar?, ¿con cuál tema central se relaciona la descripción protocolar? Estas categorías se establecieron ajustándose a los interrogantes planteados desde las observaciones: ¿qué piensa el docente acerca de la evaluación?, ¿para qué la usa?, ¿cuándo la usa?, ¿qué criterios tiene para hacer una evaluación? A este segundo paso correspondió un momento fenomenológico de lógica del significado. Se pasó de la esfera meramente fáctica, representada en este caso por las descripciones protocolares propiamente dichas, a la esfera eidética, representada por los temas centrales.

Tercer paso. Los temas centrales fueron agrupados por la similitud en lo que ellos trataban, surgiendo así once temas esenciales producto de la observación y 4 temas esenciales del proceso de entrevistas. Tales temas se relacionan en las tablas 1 y 2.

Tabla 1

Temas esenciales surgidos de la observación durante los momentos

\section{Temas}

\begin{tabular}{ll}
\hline 1 & Imperativo \\
2 & Intimidación \\
3 & Utilitarismo \\
4 & Solución del error \\
5 & Indagación \\
6 & Diversidad evaluativa \\
7 & Trabajo colectivo \\
8 & Práctica del saber \\
9 & Auto solución \\
10 & Autoritarismo \\
\hline 11 & Posibilidad \\
\hline
\end{tabular}


Tabla 2

Temas esenciales surgidos durante la entrevista

\section{Temas}

1. Aplicación instrumental

2. Enfoques

3. Dispositivo

4. Propósito
Cuarto paso. En este paso, se buscaron esencias de significado en los once y cuatro temas esenciales.

Para esto, los temas fueron reagrupados por la similitud de contenido, surgiendo así 14 categorías fenomenológicas, a las que se les dio la denominación de categorías fenomenológicas esenciales individuales sintetizadas. Ellas representan, en efecto, la síntesis que por similitud se hizo de los 15 temas esenciales. A este paso correspondieron dos momentos fenomenológicos: lógica del significado (empleo de la lógica para llegar ahora a categorías fenomenológicas esenciales sintetizadas, a partir de temas esenciales) y constitutivo trascendental (búsqueda de correspondencia entre los temas esenciales, a través de un proceso de comparación o establecimiento de semejanzas y diferencias entre los temas esenciales).

Tabla 3

Temas esenciales, categorías fenomenológicas esenciales individualizadas/ sintetizadas y categorías universales

\begin{tabular}{|c|c|c|}
\hline Categoría & $\begin{array}{c}\text { Categorías fenomenológicas/ } \\
\text { esenciales/individualizadas/sintetizadas }\end{array}$ & $\begin{array}{c}\text { Categorías fenomenológicas/ } \\
\text { esenciales/universales }\end{array}$ \\
\hline 1. Imperativo & $\begin{array}{l}\text { 1.Mandato } \\
\text { 2.Dominante }\end{array}$ & Enunciaciones autoritarias \\
\hline 2. Intimidación & $\begin{array}{l}\text { 1.Control de acciones } \\
\text { 2.Normatividad } \\
\text { 3.Imposición de ideas } \\
\text { 4.Enunciados imperativos }\end{array}$ & Dispositivo de poder \\
\hline 3. Utilitarismo & 1.Interés de resultado & Valoración de la inmediatez \\
\hline
\end{tabular}


4. Solución del error

5. Indagación

6.Diversidad

evaluativa

7. Trabajo colectivo

8. Práctica del saber

9.Autosolución

10. Autoritarismo

11. Posibilidad

12. Instrumentalización

13. Enfoques
1. Percatarse

2.Atención

1. Dudar

2. Método

3.Entender

1. Uso de las tecnologías

2.Exclusión

1. Aprendizaje colectivo

2.Resultados y trabajo de equipo

1.Pragmatismo 2. Validez de

la práctica

1.Búsqueda y dificultad

1.Examen

2.Control

1. Niega la corrección

2.Determinación

3.Suposición

1. Verificar la apropiación delconocimiento

2. Instrumento demedición

3. Indagación delaprendizaje

4.Respuesta delestudiante

5. Verificar el logro los objetivos

1. Los objetivos

2. Los saberes y presaberes

3.Conocimientosgenerales

4. Aplicación de losconocimientos

5. Conceptosbásicos.

6. Destrezas.

7. Contenidos básicos

8. Lacontextualización

9. El análisis

10. Formaciónintegral

11. Loaprendido
Organización del pensar

Tecnología del pensar

Novedad e imperativo

Hábitos deseables

Verdad y experiencia

Procedimiento

Tecnología de control

Acontecimiento

Intencionalidad /

valoración

Diversidad

\section{Dispositivo}
1. Porcompetencia
2. Escritas
3. Talleres
4. Observando laactitud
5. Evaluacionesvirtuales
6. Trabajo en elaula

Medios de Valoración 


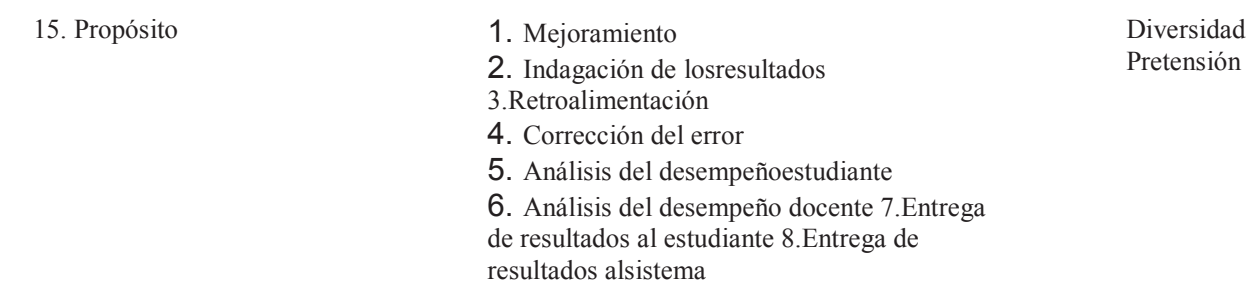

Fuente: Autor, Proceso de investigación.

Quinto paso: Las categorías fenomenológicas/ esenciales/individuales/sintetizadas, por aspectos, fueron nuevamente agrupadas por su similitud, surgiendo así 15 categorías superiores, que acá denominamos categorías fenomenológicas/ esenciales/universales. Tales categorías se reflejan en la tabla 3.

Los momentos fenomenológicos que correspondieron a este paso fueron lógica del significado (empleo de la lógica para llegar a categorías fenomenológicas/esenciales/ universales); constitutivo trascendental (búsqueda y establecimiento de correspondencia entre las categorías fenomenológicas/esenciales/ sintetizadas, para construir categorías fenomenológicas

/Esenciales /universales) y metafísico de la conciencia (comprensión de la conciencia del docente como un todo o estructura global de significado).
Por consiguiente, las categorías a las que dio lugar el despliegue del método fenomenológico seguido no describen el elemento fáctico del cual ellas emergen, en concordancia con los principios posibilitadores.

En su proceso de constitución, estas categorías fenomenológicas permitieron, como expresión del mismo método, unir, conectar, conformar, enlazar, vincular y relacionar esencias primarias de significado representadas en primera instancia por los temas centrales, a partir de los cuales y mediante una abstracción cada vez mayor, se llegó a unas categorías fenomenológicas universales, colocando entonces al investigador, al discurso de esta investigación y al sujeto que lo aborda en su lectura, lejos, distante de elementos psicofísicos, primarios, para lograr ver una totalidad, una estructura esencial representada en este caso por la conciencia del docente, lo cual se muestra en la tabla 4.

Tabla 4

Categorías relacionadas con la estructura de la conciencia del docente

Elementos estructurales

\begin{tabular}{ll}
\hline 1. & Enunciaciones autoritarias \\
2. & Dispositivos de poder \\
3. & Valoración inmediata \\
4. & Organización del pensar \\
5. & Tecnología del pensar \\
6. & Novedades e imperativos
\end{tabular}




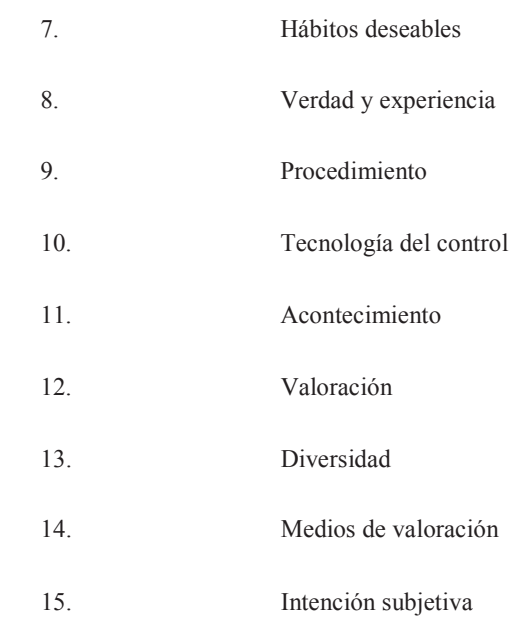

Fuente: Autor, Proceso de investigación.

\section{Discusión}

Al analizar los elementos estructurales que constituyen la conciencia del docente de la Universidad Francisco de Paula Santander, según el trabajo realizado, se encuentra que el discurso y la práctica evaluativa están enmarcados en enunciaciones de tipo autoritario, utilizado como dispositivos de poder, lo cual define el rumbo o utilización que el sujeto evaluador hace de la evaluación.

Así, se puede inferir que la conciencia de los se proyecta a través de la evaluación mostrándose en las mismas consecuencias soportadas en la exclusión, el poder, la limitación, la diferencia, la negación, la selectividad. De este modo, la conciencia trasciende, proyectándose no solo en el sujeto evaluador si no en las acciones institucionales que acogen una determinada manera de pensar.

Los resultados obtenidos mediante los elementos estructurales que constituyen la conciencia del docente de la Universidad Francisco de Paula Santander considerados en la investigación, analizados desde la visión existencialista de Sartre, resaltan lo relacional de la conciencia del docente evaluador. Estos resultados guardan relación con los hallazgos de Díaz Álvarez (2013), unos sustentados en el poder y otros en la obediencia, unos en la verdad absoluta de la evaluación otros en el resultado excluido de la misma, unos ante el determinismo de los enunciados y las acciones y otros sujetos al temor de los procedimientos, unos que normalizan los espacios de la evaluación y otros que se someten al ritual con resignación.

Los actos que llevan a cabo los docentes universitarios, en especial en aquellas acciones como notas apreciativas, lo constituyen tanto psíquica como corporalmente, siendo este un hecho irreflexivo, es decir no supone una toma de conciencia respecto a la valoración buena o mala del acto. Sin embargo cuando este acto es valorado o visto por otro, entonces ese acto se objetiva, dado que es la mirada del otro la queda consistencia a su ser. Por ello los docentes son tan esquivos a las evaluaciones alternativas (Co evaluación y Hetero evaluación).

Así se puede inferir que la conciencia de los docentes se proyecta a través de la evaluación mostrándose en las mismas consecuencias soportadas en la exclusión, el poder, la limitación, la diferencia, la negación, la selectividad. De esta manera, la conciencia trasciende proyectándose no solo en el sujeto evaluador si no en las acciones institucionales que acogen una determinada manera 
de pensar y van configurando su discurso (Kriegel, 2009).

Este discurso impuesto por los sujetos evaluadores impone grados de temor. El sujeto evaluador cree aún que el otro se debe someter por el simple hecho de pronunciar el discurso de poder. Un discurso que se impone de esta manera no establece su fuerza en lo que se hace sino en lo que se dice y no solo en lo que dice sino en la forma como se enuncia en el aula. La institución educativa crea para su aceptación un sistema de verdades que se codifica en los manuales y se revela como absoluto.

En el campo de la evaluación, el discurso evaluativo señala la forma particular como el sujeto produce los enunciados: imperativos, órdenes, formas que indican obediencia, organización, autoridad (Foucault, 1976).

Una verdad del discurso evaluativo inherente al docente es su exagerado deseo de controlar los espacios donde se ejecuta el acto pedagógico, en especial el momento de la evaluación, cuya intención es regular una serie de comportamientos que favorezca el dominio de la clase (Foucault, 1970).

Pero el control le concede al docente evaluador extraños poderes: como el de enunciar una verdad absoluta, el de predecir y definir el porvenir de otros sujetos, el de revelar desde la ingenuidad lo que la ignorancia de los otros no puede percibir.

\section{Conclusiones}

El docente de la Facultad de Ciencias Agrarias y del Ambiente expresa desde la evaluación, acciones y formas de pensar originadas desde su conciencia, en la cual se integran elementos estructurales derivados no solo de la interacción que el docente ha tenido con la evaluación si no de la transformación que se ha dado en su conciencia debido a los continuos cambios cognitivos, actitudinales, emocionales y conductuales.

El consenso que se presenta en las respuestas obtenidas de los docentes acerca de la instrumentalización de la evaluación refleja las experiencias que permitieron la constitución de ese sujeto docente, el cual reproduce los esquemas de evaluación derivadas de un discurso epocal que impone maneras de pensar y de actuar.

Las acciones más significativas identificadas en la práctica evaluativa de los docentes participantes del estudio están relacionadas con las enunciaciones autoritarias, el deseo de subordinar al sujeto evaluado, la valoración inmediata, la preservación del ritual como principio de restricción y la instrumentalización de la evaluación como dispositivo de control y de poder (Perassi y Vitarelli, 2008).

Se evidencia la condescendencia del sujeto evaluador que en algunos casos solo es parte de la ritualización de la práctica evaluativa (García, 2001).

Pero también la organización del pensar, reconocer las falencias del estudiante ayudarle a superarlas, restituir el derecho a aprender y evitar la exclusión, es una práctica que se evidencia en algunos de los docentes.

Valorar el trabajo colectivo, es una práctica que se estimula por parte de algunos de los docentes.

Las recomendaciones como trabajo complementario incluyen la comparación de los resultados de los trabajos realizados a partir de la concepción que tienen los estudiantes respecto a la evaluación y la concepción de los docentes universitarios.

La socialización de los resultados a la comunidad universitaria de Universidad Francisco de Paula Santander de los trabajos realizados en el tema de evaluación sería un aporte importante en el proceso de mejoramiento académico y acreditación institucional.

\section{Referencias}

Aguirre García, J. C. y Jaramillo Echeverri, L. G.(2012). Aportes del método fenomenológico a la investigación educativa. Revista latinoamericana de estudios educativos, 8(2): 51-74. 
Anzola, J. M. (2007). La fenomenología de la conciencia en E. Husserl.UniversitasPhilosophia, 48(24), 1127-148.

Careaga, A. (2001). La evaluación como herramienta de transformación de la práctica docente. Educere, 5(15), 345-352.

Díaz Álvarez, L. I. (2013). Fenomenología de la conciencia del docente frente a la práctica evaluativa. San José de Cúcuta: Universidad Francisco de Paula Santander.

Fermoso, P. (2008). Modelo fenomenológico de investigación en pedagogía social. Educar, 26(2), 409-430.

Foucault, M. (1970). El orden del discurso. Barcelona: Tusquets.

Foucault, M. (1976). Vigilar y castigar. México D.F.: Siglo XXI.

García, P. G. (2001). El ritual como forma de adoctrinamiento. Gaceta de Antropología, 18: 1-12. Disponible en http://www.ugr.es/ p pwlac/ G18_01Pedro_Gomez_Garcia.pdf

Lambert, C. (2006). Edmund Husserl: la idea de la fenomenología. Teología y vida, 517-529.

Martínez M. (2004). La investigación cualitativa etnográfica en educación. Manual Teórico Práctico. Buenos Aires: Trillas.

Moustakas, C. (1994). Phenomenological research methods. Thousand Oaks. Calif. SAGE.

Mollà, R. M., Bonet, R. M. B., yCliment, C. I. (2010). Propuesta de análisis fenomenológico de los datos obtenidos en la entrevista. UniversitasTarraconensis. Revista de Ciències de l'Educació, 1(1), 113-133.

Torres, M. F. (2010). ¿Qué y cómo se ha investigado sobre la evaluación de los aprendizajes en los últimos años? Estado del arte de las investigaciones. Enunciación, 15(1): 145-160.

Kriegel, U. (2009). Teorías de la conciencia. Praxis filosofica, 29: 178-188.

Perassi, Z. y Vitarelli, M. (2008). Evaluación y práctica docente: su impacto en el campo educativo. Disponible en http://www.uccor.edu. ar/paginas/encuentros2005/VitarelliPerassiI.pdf

Weeks, D.R. (1984), Las organizaciones:
Interacción y procesos sociales. En G. Salman y K. Thompson.Control e ideología en las organizaciones México D.F.: Fondo de Cultura Económica, pp. 123-147.

Zuluaga, C. F. (2009). Sentido de las prácticas evaluativas. Trabajo de grado no publicado. Manizales: Universidad de Caldas. 